\title{
A Propose Model Optimal Supply Chain Distribution Network for Farmer Industrial
}

\author{
Ammar Salamh Mujali Al-Rawahna ${ }^{1, *}$, Anas Yahya Bader Al Hadid ${ }^{2}$ \\ ${ }^{1}$ Southern Taiwan University of Science and Technology, Taiwan \\ ${ }^{2}$ Amman Arab University, Jordan \\ ${ }^{1}$ rawahna2007@gmail.com \\ * corresponding author
}

(Received August 25, 2020 Revised September 9, 2020 Accepted September 19, 2020, Available online September 30, 2020)

\begin{abstract}
A supply chain should be operated in the most efficient way in a highly competitive environment, with the goals of cost minimization, shipment delays, inventories and expenditures, and distribution maximization, gain, return on investment, level of customer support, and efficiency. The development of supply-chain distribution networks is therefore an extremely complex task, due to the large physical production and distribution network flows, the uncertainties associated with external interface customers and suppliers as well as the non-linear dynamics linked to internal information flows. This study aims to address a problem in domestic distribution in a supply chain system that includes manufacturers, distribution centers and consumer zones to determine the optimum configuration of the network. We propose a mixed integer linear programming model to solve the problem.
\end{abstract}

Keywords: Optimization; Mixed Integer Programming; Linier Programming; Distribution Network; Supply Chain Management.

\section{Introduction}

Organizations must ensure that resources are used effectively in a highly competitive environment. To this end, supply chain management must concentrate on efficient actors being successfully integrated. This topic has been intensively investigated and various approaches can be used. For example, see the survey of resource choice (productive actors) provided by Pires et al [7] The relationship between tactical, organizational and strategic decisions is also important in supply chain management.

Throughout today's market, the role of supply chain management (SCM) is becoming increasingly important. The researchers have shifted to an integrated and strategic approach through a purely operational approach to SCM in the sixties. Today, SCM is regarded as a source of skill and innovation. The management of supply chains is nonetheless a very complex task because of the large physical production and distribution network channels, inconsistencies related to existing consumer and distributor interfaces and nonlinear information flows.

The selection of best locations in warehouses or distribution centers (DC's), which can result in significant cost savings due to reductions in transport or shipping costs, is an important strategic concern for the planning and running of physical distribution networks in a supply chain system. The problem is "How many DCs should we have? And "Where should they be allocated?" This is one of the most complex challenges companies are facing today: the choice of the network for distribution. When faced with cost-reduction pressures while increasing service levels for customers, this challenge is stepped up.

The problem of developing a distribution network involving the determination of the optimum number and best positions of DCs and the optimal approach to transport the material from the plants to the DCs and from the DCs to the client areas is addressed in this study. We are proposing a mathematical model for the development of the supply chain networks, combining output, material handling and transport components. The network comprises production facilities, DCs and customer areas. The plants and consumer areas have already been built at certain sites. There are already distribution facilities at certain locations and consumer 
regions. The location of the DCs is chosen from the nominee places. As part of the optimization problem, the layout and connectivity of each DC are considered.

\section{Literature Review}

The key issues in contemporary supply chain management consist of distribution network configuration, and distribution strategies, etc., as Simchi-Levi et al [9] mentioned. The distribution network configuration deals with the design of a distribution network to serve a specific market. This will consist of a set of warehouses and retail outlets, together with the manufacturing plant and supply sources.

Distribution refers to the steps taken to move and store a product from the supplier stage to a customer stage in the supply chain, as Chopra and Meindl [4] defined. In the supply chain distribution occurs between every pair of stages. Because distribution affects both the supply chain cost and the customer experience directly, it is a key driver of the overall profitability of a firm.

At the highest level, performance of a distribution network should be evaluated along two dimensions: customer needs that are met, and cost of meeting customer needs. Consequently, a firm must estimate the impact on customer service and cost as it compares different distribution network options. The customer needs that are met impact the company's revenues, which along with cost decide the profitability of the delivery network.

Geoffrion and Graves [5] presented a model to solve the problem of designing a distribution system with optimal location of the intermediate distribution facilities between plants and customers. The objective is to minimize the total distribution cost, subject to a number of constraints such as supply constraints, demand constraints, and specification constraints regarding the nature of the problem. The problem is formulated as an MILP, which is solved using Benders decomposition.

Brown et al [3] presented an optimization-based algorithm for a decision support system used to manage complex problems involving facility selection, equipment location and utilization, and manufacture and distribution of products. They focus on operational issues such as where each product should be produced, how much should be produced in each plant, and from which plant products should be shipped to customers.

Arntzen et al [1] developed an MILP "global supply chain model" aiming to determine: (1) the number and location of DCs, (2) customer-DC assignment, (3) number of echelons, and (4) the product-plant assignment. The objective of the model is to minimize a weighted combination of total cost (including production, inventory, transportation, and fixed costs) and activity days.

In a later work, Pirkul et al [8] presented the PLANWAR model that seeks to locate a number of production plants and DCs so that the total operating cost for the distribution network is minimized. The network consists of a potential set of production plants and DCs and a set of given customers.

Tsiakis et al. [11] had addressed a large number of the production and logistics issues reported by Bilgen [2] in a comprehensive work that models supply chain networks under demand uncertainty focusing on the production and transportation issues. The resulting MILP problem is solved to optimality using decomposition methods to reduce the computational effort required.

In the later work, Tsiakis and Papageorgiou [10] determined the optimal configuration of a production and distribution network subject to operational and financial constraints. Out-sourcing of production is considered in case the organization cannot satisfy the customer demand. A MILP model proposed to describe the optimization problem is a global supply chain model emphasizing on production activities with demand certainly. 
Bilgen addressed the production and distribution planning problem in a supply chain system that involves the allocation of production volumes among the different production lines in the manufacturing plants, and the delivery of the products to the DCs. As a real supply chain operates in a highly dynamic and uncertain environment, this model was transformed into fuzzy models taking into account the fuzziness in the capacity constraints, and the aspiration level of costs using different aggregation operators [12].

\section{Method}

In this section, a mathematical model will be presented to solve the distribution issue in a domestic supply chain network. The above problem is formulated mathematically as an MILP optimization problem. We aim to obtain an optimal design of this network.

\subsection{Notation, Parameter and Variables}

Indices/sets:

\section{j Production plants}

$k \quad$ Possible distribution centers

$l \quad$ Customer demand zones

Parameters:

$C_{k}^{D, e} \quad$ Annualized fixed cost of establishing a distribution center at location $k$

$C_{j}^{P} \quad$ Unit production cost at plant $j$

$C_{k}^{D, H} \quad$ Unit handling cost for product at distribution center $k$

$C_{j k}^{T} \quad$ Unit transportation cost for product from a plant $j$ to a distribution center $k$

$C_{k l}^{T} \quad$ Unit transportation cost for product from a distribution center $k$ to a customer zone $l$

$D_{l} \quad$ Demand for product in a customer zone $l$

$D_{k}^{\operatorname{Min}}, D_{k}^{\operatorname{Max}} \quad$ Minimum/maximum distribution center capacity

$P_{j}^{\text {Min }}, P_{j}^{\text {Max }} \quad$ Minimum/maximum production capacity of plant $j$

$Q_{j k}^{\operatorname{Min}}, Q_{j k}^{\operatorname{Max}} \quad$ Minimum/maximum quantity of products shipped from a plant $j$ to a distribution center $k$

$Q_{k l}^{M i n}, Q_{k l}^{\operatorname{Max}} \quad$ Minimum/maximum quantity of products shipped from a distribution center $k$ to a customer zone $l$

Continuous variables:

$D_{k} \quad$ Capacity of a distribution center $k$

$P_{j} \quad$ Production rate in plant $j$

$Q_{j k} \quad$ Quantity of product shipped from plant $j$ to a distribution center $k$

$Q_{k l} \quad$ Quantity of product shipped from distribution center $k$ to a customer zone $l$ 
Binary variables:

$Y_{k} \quad$ A binary variable; 1 if distribution center $k$ is to be established, 0 otherwise

$X_{j k} \quad$ A binary variable; 1 if production plant $j$ is assigned to distribution center $k, 0$ otherwise

$X_{k l} \quad$ A binary variable; 1 if distribution center $k$ is assigned to customer zone $l, 0$ otherwise

\subsection{Problem Definition}

This research considers the optimal design and operation of a distribution supply chain network that consist of one product type. The network involves a number of existing manufacturing sites at fixed locations, a number of DCs of unknown locations (to be selected from a set of candidate locations), and a number of customer zones at fixed locations.

Generally, the product can be produced at all plants at different locations. A DC can be supplied from more than one manufacturing plant, and can supply more than one customer zone. Each customer zone places demands for the product and can be served by more than one DC.

In case of "single sourcing" constraints, according to which a DC must be supplied from one manufacturing site, and a customer zone must be served by a single DC, may be performed by modifying the constraints.

The production capacity of each manufacturing site is modeled in terms of a set of linear constraints relating the mean production rate of product to the availability of the plant. DCs are described by upper and lower bounds on their material handling capacity. Operational costs include those associated with production, handling of material at DCs and transportation.

The decisions to be determined include production amounts of product, the number, location, and capacity of DCs to be setup, the flows of product, and transportation links to establish in the network. The objective is to minimize the total annualized cost of the supply chain network.

The network illustrated in Figure 1 presents the configuration in which $n$ production plants, $m$ DCs, $p$ customer zones and its possible connections exist.

The network design model presented in this research is a simplified model of Tsiakis et al [11] by emphasizing on flow of product and transportation cost in distribution network.

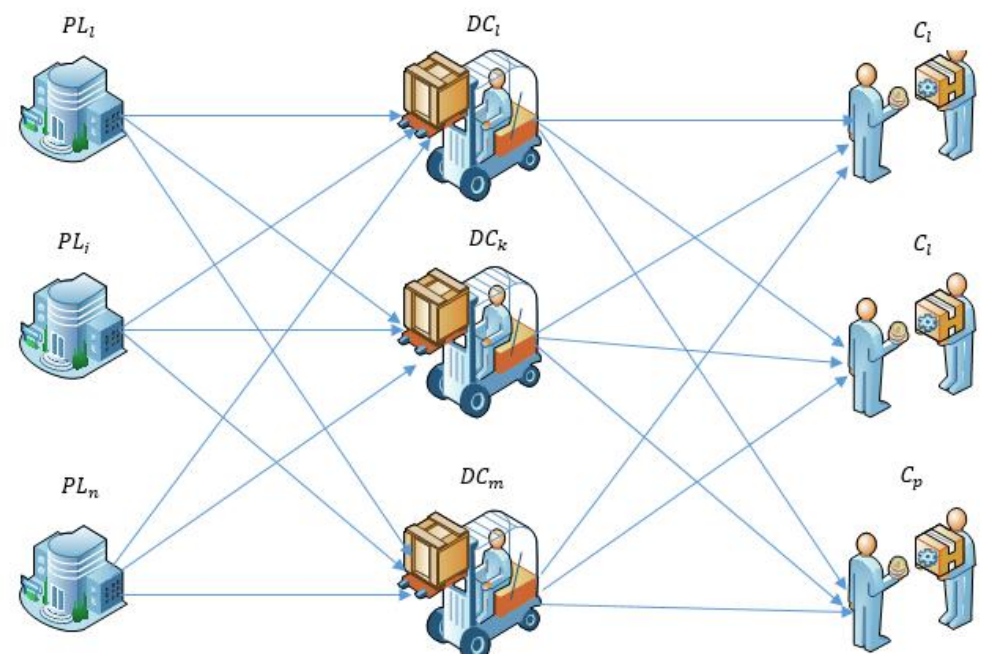

Fig. 1. Network configuration illustration 


\subsection{Problem Assumption}

Because of our research limitation, a number of assumptions are considered to make a validity of the model. The major assumptions are described as follows:

1. The supply network consists of several existing plants which deliver the final product to various DCs.

2. The problem is considered as a steady-state form, or in other words there is no stock accumulation or depletion.

3. Production and transportation flow are considered to be time-averaged quantities.

4. Customers' demands are time-invariant and given.

5. Transportation costs are linear function of the flow of product in distribution network.

6. The capacities of the DCs are related linearly to the flows of materials that they handle.

\subsection{Constraint}

This section presents several constraints needed to determine a network configuration.

a. Network Structure Constraint

A link between a production plant $j$ and a DC $k$ may exist only if the production plant $j$ also exists. In other words, the production plant $j$, in case of its establishment, can whether supply to a DC $k$. This constraint is presented by the attribute of binary variable $X_{j k}$.

If a DC $k$ is established, it can then be served by more than one production plant $j$ :

$$
\sum_{j} X_{j k} \geq Y_{k}, \forall k
$$

In accordance with the "single sourcing", this constraint has to be modified as:

$$
\sum_{j} X_{j k}=Y_{k}, \forall k
$$

The link between a DC $k$ and a customer zone $l$ may exist only if the DC $\mathrm{k}$ is established. In other words, if a DC $k$ exists, it can whether supply to a customer zone $l$ :

$$
X_{k l} \leq Y_{k}, \forall k, l
$$

Each customer zone can be supplied by more than one single DC to satisfy its demand:

$$
\sum_{k} X_{k l} \geq 1, \forall l
$$

In case of "single sourcing", this constraint has to be written as:

$$
\sum_{k} X_{k l}=1, \forall l
$$

b. Logical Constraint for Transportation Flows

Flow of product from production plant $j$ to DC $k$ can take place only if the corresponding connection exists:

$$
Q_{j k}^{M i n} X_{j k} \leq Q_{j k} Q_{j k}^{\operatorname{Max}} X_{j k}, \forall j, k
$$


Flow of material from DC $k$ to customer zone $l$ can take place only if the corresponding connection exists:

$$
Q_{k l}^{\operatorname{Min}} X_{k l} \leq Q_{k l} Q_{k l}^{\operatorname{Max}} X_{k l,}, \forall k, l
$$

\section{c. Material Balances}

The actual rate of production by plant $\mathrm{j}$ must equal the total flow of this product from plant $j$ to all DCs $k$ :

$$
P_{j}=\sum_{k} Q_{j k}, \forall j
$$

Because of steady-state form, according to which there is no stock accumulation or depletion, the total rate of flow of product leaving a DC must equal the total rate of flow entering this node of the supply chain network:

$$
\sum_{j} Q_{j k}=\sum_{l} Q_{k l}, \forall k
$$

Ideally the total rate of flow of product received by each customer zone 1 from the DCs must be equal to the corresponding market demand:

$$
\sum_{k} Q_{k l}=D_{l}, \forall l
$$

\section{d. Production Constraint}

The rate of production of product at any plant cannot exceed certain limits. Thus, there is always a maximum production capacity for any product; moreover, there is often a minimum production rate that must be maintained while the plant is operating:

$$
P_{j}^{M i n} Y_{j} \leq P_{j} \leq P_{j}^{\operatorname{Max}} Y_{j}, \forall j
$$

\section{e. Capacity of DCs}

In case a DC k is established, its capacity has to be lie between the lower and upper bounds. This constraint is described as follow:

$$
D_{k}^{\text {Min }} Y_{k} \leq D_{k} \leq D_{k}^{\operatorname{Max}} Y_{k}, \forall k
$$

We generally assume that the capacities of the DCs are related linearly to the flows of materials that they handle. This is expressed via the constraints:

$$
D_{k} \geq \sum_{l} Q_{k l}, \forall k
$$

\subsection{Objective Function}

In general, costs of a distribution network involves those of fixed infrastructure, production of material at plants, the handling of material at DCs, and the transportation of material through the network. 


\section{a. $\quad$ Fixed Infrastructure Cost}

The infrastructure cost considered by our formulation is related to the establishment of a DC at a candidate location. This cost can be express as follow:

$$
\sum_{k} C_{k}^{D, e} Y_{k}
$$

The production plants are assumed to be already established. Therefore, the capital cost associated with their design and construction is not to be considered. Any infrastructure cost associated with the customer zones is also ignored.

\section{b. Production Cost}

The production cost is given by the production rate $P_{j}$ of product in plant $\mathrm{j}$, with the unit production $\operatorname{cost} C_{j}^{p}$. The unit production cost $C_{j}^{p}$ is expressed in terms of local currency. The production cost corresponding in the objective function is formed as follow:

$$
\sum_{j} C_{j}^{p} P_{j}
$$

c. Material Handling Cost at DCs

Handling costs can usually be approximated as linear functions of the total throughput. They can be expressed as follows:

$$
\sum_{k} C_{k}^{D H}\left(\sum_{l} Q_{k l}\right)
$$

\section{d. Transportation Cost}

The unit transportation cost is constant independent of the product transported and depends only on the source and destination node. Transportation costs that we include are those between production plant to DCs and DCs to customers. Therefore the following term needs to be added to the objective function:

$$
\sum_{j, k} C_{j k}^{T} Q_{j k}+\sum_{k, l} C_{k l}^{T} Q_{k l}
$$

\section{e. Overall Objective Function}

By combining the cost terms derived from Sections 3.5.1 to 3.5.4, we obtain the total cost of the supply chain distribution network, which is to be determined by the optimization:

$$
\operatorname{Min} Z=\sum_{k} C_{k}^{D, e} Y_{k}+\sum_{j} C_{j}^{p} P_{j}+\sum_{k} C_{k}^{D H}\left(\sum_{l} Q_{k l}\right)+\sum_{j, k} C_{j k}^{T} Q_{j k}+\sum_{k, l} C_{k l}^{T} Q_{k l}
$$

\section{Case Study and Computation Result}

\subsection{Model Verification}

To value preliminary the effectiveness of mathematical model and the validity of LINGO program, an example developed by Geoffrion and Van Roy [6] is considered. 


\section{a. Example Description}

The distribution system in this example existed with the following traits:

1. Single product

2. Two plants, referred to as plant $p 1$ and plant $p 2$.

3. Plant $p 2$ has an annual capacity of 60,000 units.

4. The two plants have the same production costs.

5. Two existing warehouses, referred to as warehouse $w 1$ and warehouse $w 2$, have identical warehouse handling costs.

6. Three market areas, $c 1, c 2$, and $c 3$, with demands of 50,000, 100,000 and 50,000, respectively.

The distribution cost per unit is given in Table 1 .

Table. 1. Distribution Cost Per Unit

\begin{tabular}{|c|c|c|c|c|c|}
\hline \multirow{2}{*}{ Warehouse } & \multicolumn{2}{|c|}{ Plant } & \multicolumn{3}{c|}{ Market Area } \\
\cline { 2 - 6 } & $\mathrm{p} 1$ & $\mathrm{p} 2$ & $\mathrm{c} 1$ & $\mathrm{c} 2$ & 5 \\
\hline $\mathrm{w} 1$ & 0 & 4 & 3 & 4 & 2 \\
\hline $\mathrm{w} 2$ & 5 & 2 & 2 & 1 & 5 \\
\hline
\end{tabular}

Our objective is to find an optimal distribution strategy that specifies the flow of products from the suppliers through the warehouses to the market areas without breaking the plant $\mathrm{p} 2$ production capacity constraint, that satisfies market area demands, and that minimizes total distribution costs.

Two intuitive heuristics can be considered in order to solve this problem. In the first heuristics, the cheapest warehouse to source demand is chosen for each market and $\$ 1,120,000$ is the result of total distribution costs. In the second way, the warehouse where the total delivery costs to and from the warehouse are the lowest, is chosen for each market. The total distribution costs for this strategy is $\$ 920,000$.

However, those two heuristics do not generate the best strategy for this distribution system. To find the best strategy, an optimization model by framing the distribution problem as linear programming problem is provided.

For this purpose, flows of product from the plants to the warehouses and from warehouses to customer zones are the continuous variables denoted by $x(p j, p k)$ and $x(p k, p l)$, respectively, while $j, k$, and $l$ are the index set of plants, warehouses and customer zones. The linear programming problem we need to solve is:

The objective function:

$$
\operatorname{Min} \mathrm{Z}=\quad \begin{aligned}
& \{0 x(p 1, w 1)+5 x(p 1, w 2)+4 x(p 2, w 1)+2 x(p 2, w 2)+3 \\
& x(w 1, c 1)+4 x(w 1, c 2)+5 x(w 1, c 3)+2 x(w 2, c 1)+1 x(w 2, \\
& c 2)+2 x(w 2, c 3)\}
\end{aligned}
$$

Subject to the following constraints:

$$
\begin{aligned}
& x(p 2, w 1)+x(p 2, w 2) \leq 60,000 \\
& x(p 1, w 1)+x(p 2, w 1)=x(w 1, c 1)+x(w 1, c 2)+x(w 1, c 3)
\end{aligned}
$$




$$
\begin{aligned}
& x(p 1, w 2)+x(p 2, w 2)=x(w 2, c 1)+x(w 2, c 2)+x(w 2, c 3) \\
& x(w 1, c 1)+x(w 2, c 1)=50,000 \\
& x(w 1, c 2)+x(w 2, c 2)=100,000 \\
& x(w 1, c 3)+x(w 2, c 3)=50,000
\end{aligned}
$$

Using the Excel linear programming solver, the authors archived the value of $\$ 740,000$ as total cost for the optimal strategy.

\section{b. Mathematical Model Verification}

Considering above example, it is essentially a simplified case of this research problem in which the mathematical model in Section 3 must be adjusted with existing DCs. The quantities of products shipped from manufacturing plants to DCs and from DCs to customer zones are only the continuous variables. "Single sourcing" constraints are not applied in this case. Our objective is to find the strategy that reaches the minimum value of the transportation costs.

In this case the mathematical model is described as follow:

Objective function:

$$
\operatorname{Min} \mathrm{Z}=\sum_{j, k} C_{j k}^{T} Q_{j k}+\sum_{k, l} C_{k l}^{T} Q_{k l}
$$

subject to:

$$
\begin{aligned}
& \sum_{j} X_{j k} \geq 1, \forall k \\
& \sum_{k} X_{k l} \geq 1, \forall l \\
& Q_{j k}^{\text {Min }} X_{j k} \leq Q_{j k} Q_{j k}^{\text {Max }} X_{j k}, \forall j, k \\
& Q_{k l}^{\text {Min }} X_{k l} \leq Q_{k l} Q_{k l}^{\text {Max }} X_{k l,}, \forall k, l \\
& P_{j}=\sum_{k} Q_{j k}, \forall j \\
& \sum_{j} Q_{j k}=\sum_{l} Q_{k l}, \forall k \\
& \sum_{k} Q_{k l}=D_{l}, \forall l \\
& P_{j}^{M i n} Y_{j} \leq P_{j} \leq P_{j}^{M a x} Y_{j}, \forall j
\end{aligned}
$$

The objective value $\$ 740,000$ archived after running LINGO program is the same with the result previously provided by the authors. 
Moreover, the LINGO computation results also provided the equally distribution strategy. Thus, we can conclude that the mathematical model presented in this research is suitable for the case, and LINGO program that we made can solve this problem.

\subsection{Case Study}

In this section, an industrial case study will be presented to illustrate the mathematical model applicability.

$\mathrm{ABC}$ is a manufacturer operating in a domestic chemical industry. It has three manufacturing plants producing one family of product (now we call one product), and located in three different areas. Product demands can be divided into five customer zones located in different locations close to the DCs. A number of possible DCs are considered to handle the material based on the market demand placed by the customer zones. The network structure which assumes complete connectivity between all sites is given in the Figure 2 .

A scenario in which all decisions are determined by the model subject to the constraints is examined to demonstrate the benefits of $\mathrm{ABC}$ optimization distribution network. The "single sourcing" constraint which considers the case where each customer zone is served by one specific DC is applied.

\section{Conclusion and Recommendation Future Research}

\subsection{Conclusion and Contribution}

Due to the inherent complexity of the major subsystems of these networks and the many interactions between these subsystems, the development of distribution supply chain networks is a difficult task. The complexity in this area has forced a large part of research in the past to focus on individual supply chain network components. Over recent times, however, the efficiency, design and evaluation of the supply chain as a whole have become increasingly attentive.

We propose in this research a model based on a developed model to tackle a complexity of the abovementioned problem. The model takes into account the problem of designing a distribution network on a supply chain system to determine the best locations of DCs and the best strategy to supply the commodity via a network.

The study allows for the design of a distribution network suited to domestic companies like farmer producers. In contrast to previous investigations. We help to determine a MILP model that is appropriate for the layout of the network by reducing the overall annualized supply chain costs, and deciding which factories, DCs and how much they are transported to meet the needs of the company.

\subsection{Recommendation for Further Research}

The model described in this research specifically takes into account either one product category or one product. For further analysis, the index of the continuous variables: $j, Q_{j k}$ and $Q_{k l}$ can be extended to cover multiple products, and adjusted corresponding constraints accordingly.

Furthermore, the manufacturing plants are modeled by their full capacity to make the product. In practice, designing a production plant with production lines to reduce unit cost of production is realistic. In addition, in the case of multiple products or product groups, due to the high investment costs, it is often not feasible to install production equipment for the entire product range at each factory. Thus, designing a number of production lines can be considered as one of the optimization solution in each manufacturing plant. Each production line can be considered as a single stage process at each individual plant capable of producing several production groups. 
Another way to develop the model can be concerned is to use multiple levels of capacity for each facility. Using different levels of capacity makes the problem more realistic and more complex to solve at the same time.

\section{References}

[1] B. C. Arntzen, G. G. Brown, T. P. Harrison, and L. L. Trafton, "Global Supply Chain Management at Digital Equipment Corporation,” Interfaces (Providence)., vol. 25, no. 1, pp. 69-93, 1995, doi: 10.1287/inte.25.1.69.

[2] B. Bilgen, "Application of fuzzy mathematical programming approach to the production allocation and distribution supply chain network problem," Expert Syst. Appl., vol. 37, no. 6, pp. 4488-4495, 2010, doi: 10.1016/j.eswa.2009.12.062.

[3] G. G. Brown, G. W. Graves, and M. D. Honczarenko, "Design and Operation of a Multicommodity Production/Distribution System Using Primal Goal Decomposition.," Manage. Sci., vol. 33, no. 1, pp. 1469-1480, 1987, doi: $10.1287 / \mathrm{mnsc} .33 .11 .1469$.

[4] P. Chopra, Sunil,. Meindle, "Supply Chain Mangement Strategy , Planning, and Operation," J. Chem. Inf. Model., pp. 1-14, 2001, doi: 10.1017/CBO9781107415324.004.

[5] A. M. Geoffrion and G. W. Graves, "Multicommodity Distribution System Design," no. August 2010, 2010, doi: 10.1007/978-1-4419-6810-4.

[6] Geoffrion, A. M., \& Van Roy, T. J. (1979). Caution: Common Sense Planning Methods Can Be Hazardous to Your Corporate Health. Sloan Management Review, 20(4), 31-42.

[7] A. Pires, G. Putnik, and P. Ávila, "A survey analysis of the resource selection models in Agile/Virtual Enterprises," J. Appl. Res. Technol., vol. 10, no. 3, pp. 416-427, 2012, doi: 10.22201/icat.16656423.2012.10.3.399.

[8] H. Pirkul and V. Jayaraman, "a Multi-Commodity , Multi-Plant, Capacitated Facility Location Problem : Formulation and," Comput. Oper. Res., vol. 25, no. 10, pp. 869-878, 1998.

[9] Simchi-Levi, D., Kaminsky, P., \& Simchi-Levi, E, "Designing and Managing the Supply Chain: Concepts, Strategies, and Case Studies." McGraw-Hill. 2003.

[10]P. Tsiakis and L. G. Papageorgiou, "Optimal production allocation and distribution supply chain networks," Int. J. Prod. Econ., vol. 111, no. 2, pp. 468-483, 2008, doi: 10.1016/j.ijpe.2007.02.035.

[11]P. Tsiakis and N. Shah, "Design of Multi-echelon Supply Chain Networks under Demand Uncertainty Design of Multi-echelon Supply Chain Networks under Demand,” Ind. Eng. Chem. Res., no. JULY 2001, pp. 3585-3604, 2016, doi: $10.1021 / \mathrm{ie} 0100030$.

[12]T. Hariguna, H. T. Sukmana, and J. Il Kim, "Survey oponion using Sentiment Analysis," J. Appl. Data Sci., vol. 1, no. 1, pp. 35-40, 2020. 